\title{
Normalized Least-Mean-Square Algorithm with a Pseudo-Fractional Number of Orthogonal Correction Factors
}

\author{
Sang Mok Jung, Ji-Hye Seo, and Poo Gyeon Park
}

\begin{abstract}
This paper proposes a normalized LMS algorithm (NLMS) that automatically determines the number of orthogonal correction factors (OCFs) by using a pseudo-fractional method, which relaxes the constraint that the number of OCFs in the NLMS algorithm must be integral and introduces the concept of a pseudo-fractional OCF number in the adaptation rule. The pseudo-fractional OCF number is adjusted by using the difference between the averages of the accumulated squared-output errors. The experimental results show that the proposed algorithm has not only a fast convergence rate but also a small steady-state estimation error with low computational complexity in comparison to existing algorithms with multiple input vectors.
\end{abstract}

Index Terms-Adaptive filters, normalized LMS with orthogonal correction factors (NLMS-OCF), pseudo-fractional OCF number, pseudo-fractional method.

\section{INTRODUCTION}

The normalized LMS (NLMS) algorithm is widely used in adaptive filtering owing to its low computational complexity and ease of implementation [1], [2]. However, its convergence is deteriorated by colored input signals. To overcome this disadvantage, several algorithms such as the affine projection algorithm (APA) [3], the partial rank algorithm (PRA) [4], and the normalized LMS algorithm with orthogonal correction factors (NLMS-OCF) [5] have been developed over the decades. These algorithms update the weights on the basis of multiple input vectors, while the NLMS algorithm updates the weights on the basis of a single input vector. They achieve fast convergence rate, but have high computational complexity and a large steady-state estimation error because of the multiple input vectors.

To solve these problems, several methods have been suggested that adjust the number of input vectors in the updating procedure [6]-[8]. The APA with dynamic selection of input vectors (DS-APA) [6] and the family of APAs with dynamic selection of input vectors (DS-N-APA) [8] suggest the ideal selection criterion of the input vectors and perform

Manuscript received September 2, 2014; revised March 23, 2015. This research was supported by the MSIP (Ministry of Science, ICT and Future Planning), Korea, under the "IT Consilience Creative Program" (NIPA-2014-H0201-14-1001) supervised by the NIPA (National IT Industry Promotion Agency).

S. M. Jung and P. G. Park are with the Electrical Engineering Department, Pohang University of Science and Technology (POSTECH), Pohang, Korea (e-mail: illus2@postech.ac.kr,ppg@postech.ac.kr).

J.-H. Seo is with the Division of IT Convergence Engineering, Pohang University of Science and Technology (POSTECH), Pohang, Korea (e-mail: bmclubhs@postech.ac.kr). the updating procedure with the selected input vectors. These algorithms can achieve lower computational complexity and smaller steady-state estimation error than other existing algorithms using multiple input vectors; however, they still have a larger steady-state estimation error than the NLMS algorithm.

This paper proposes the NLMS-OCF algorithm that determines the number of orthogonal correction factors (OCFs) by employing a pseudo-fractional method that was motivated from the concept of the pseudo-fractional tap length [9]. The pseudo-fractional method relaxes the constraint on the conventional NLMS-OCF algorithm that the number of OCFs must be integral, and it determines the number of OCFs by using the difference between the averages of the accumulated squared-output errors. By using both integral and pseudo-fractional numbers of OCFs, the proposed algorithm ensures a fast convergence rate and a small steady-state estimation error. In addition, it has lower computational complexity than the existing algorithms with multiple input vectors.

\section{NORMALIZED LMS ALGORITHM WITH ORTHOGONAL CORRECTION FACTORS}

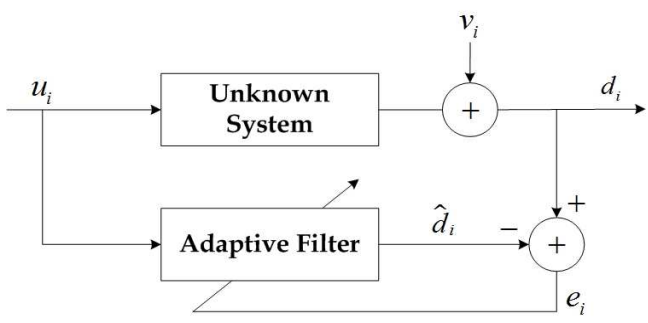

Fig. 1. Adaptive filtering structure.

Fig. 1 shows an adaptive filtering structure in the system identification application. The objective is to estimate an n-dimensional weight vector $\hat{\mathbf{w}}_{i}$ that makes the estimated error $e_{i}=d_{i}-\hat{d}_{i}$ as small as possible in the mean-squared-error sense. The estimated output and the true output can be expressed as $\hat{d}_{i}=\mathbf{u}_{i}^{T} \hat{\mathbf{w}}_{i}$ and $d_{i}=\mathbf{u}_{i}^{T} \mathbf{w}+v_{i}$, respectively, where $\mathbf{W}$ is an unknown column vector that we expect to estimate, $\mathbf{u}_{i}=\left[u_{i}, u_{i-1}, \ldots, u_{i-n+1}\right]^{T}$ denotes an $n$-dimensional input column vector at the $i$-th iteration, and $v_{i}$ accounts for measurement noise with variance $\sigma_{v}^{2}$.

The weight updating equation of the NLMS-OCF algorithm is: 


$$
\hat{\mathbf{w}}_{i+1}=\hat{\mathbf{w}}_{i}+\bar{\mu}_{0} \mathbf{u}_{i}+\bar{\mu}_{1} \mathbf{u}_{i}^{(1)}+\cdots+\bar{\mu}_{M} \mathbf{u}_{i}^{(M)}, i \geq M<n
$$

where $\mathbf{u}_{i}^{(j)}(j=1,2, \ldots, M)$ are the components of $\mathbf{u}_{i-j D}$ that are orthogonal to $\mathbf{u}_{i}, \mathbf{u}_{i-D}, \mathbf{u}_{i-2 D}, \ldots, \mathbf{u}_{i-(j-1) D}$ and can be computed using the Gram-Schmidt procedure [10], $M$ is the number of OCFs, and $D$ is the delay between the input vectors used for the updating procedure. The value of $D$ is chosen to be $\lceil M / 2\rceil$ in this paper, where the operator $\lceil$. rounds to the nearest integer towards infinity. Moreover, $\bar{\mu}_{k}(k=0,1, \ldots, M)$ is calculated according to

$$
\bar{\mu}_{k}= \begin{cases}\frac{\mu e_{i}}{\mathbf{u}_{i}^{T} \mathbf{u}_{i}} & \text { for } k=0, \text { if }\left\|\mathbf{u}_{i}\right\| \neq 0 \\ \frac{\mu e_{i}^{(k)}}{\mathbf{u}_{i}^{(k)^{T}} \mathbf{u}_{i}^{(k)}} & \text { for } k=1,2, \ldots, M, \text { if }\left\|\mathbf{u}_{i}^{(k)}\right\| \neq 0 \\ 0 & \text { otherwise }\end{cases}
$$

where $0<\mu<2$ is the step size,

$$
\begin{aligned}
& e_{i}=d_{i}-\mathbf{u}_{i}^{T} \hat{\mathbf{w}}_{i}, \\
& e_{i}^{(k)}=d_{i-k D}-\mathbf{u}_{i-k D}^{T} \hat{\mathbf{w}}_{i}^{(k)}, \text { and } \\
& \hat{\mathbf{w}}_{i}^{(k)}=\hat{\mathbf{w}}_{i}+\bar{\mu}_{0} \mathbf{u}_{i}+\bar{\mu}_{1} \mathbf{u}_{i}^{(1)}+\cdots+\bar{\mu}_{k-1} \mathbf{u}_{i}^{(k-1)}
\end{aligned}
$$

\section{Proposed Algorithm: Normalized LMS WITH PSEUDO-FRACTIONAL NUMBER OF ORTHOGONAL CORRECTION FACTORS}

In this section, we introduce the concept of a pseudo -fractional number to control the number of OCFs, $M$, in the NLMS-OCF algorithm and we propose a pseudo-fractional method based on this concept. Moreover, the entire procedure of the proposed algorithm is summarized.

\section{A. Pseudo-Fractional Method}

The performance of the NLMS-OCF algorithm is affected by the number of orthogonal correction factors. The NLMS-OCF algorithm with a large $M$ has fast convergence rate in the transient state but a large estimation error in the steady state. Conversely, the NLMS-OCF algorithm with a small $M$ converges slowly but has a small steady-state estimation error. Therefore, determining the proper value of $M$ is a very important issue when using the NLMS-OCF algorithm

Generally, the NLMS-OCF algorithm has a constraint that the value of $M$ must be integral. Because of this constraint on $M$, we cannot apply a small leaky factor in the adaptation rule for $M$. If the possible values of $M$ can be considered to include not only integers but also non-integers, we can apply the leaky factor to the adaptation rule for $M$. Based on such motivation, this paper proposes a pseudo-fractional method that introduces the concept of pseudo-fractional OCF numbers to determine $M$ by considering its properties.

The pseudo-fractional method introduces both the integral
OCF number and the pseudo-fractional OCF number. The integral OCF number is needed to perform the updating procedure in the proposed algorithm, and the pseudo-fractional OCF number is needed to obtain the integral OCF number. The integral OCF number remains unchanged until the change of the pseudo-fractional OCF number accumulates to some extent. The pseudo-fractional OCF number is obtained by comparing the averages of the accumulated squared-errors and by using the leaky factor. When the difference between the integral and pseudo-fractional OCF numbers becomes greater than a predetermined value, the integral OCF number can be obtained from the integral part of the pseudo-fractional OCF number. The proposed method dynamically adjusts $M$ and leads to the improvement of the performance in terms of the convergence rate and the steady-state estimation error.

Specifically, the pseudo-fractional OCF number at iteration $i$ is denoted by $M_{f, i}$ and is computed with the following adaptation rule:

$$
M_{f, i}= \begin{cases}\left(M_{f, i-1}-\alpha\right)- & \\ \gamma\left[A A S E_{M_{i}}-A A S E_{M_{i}-\Delta}\right], & \text { if } M_{i} \geq \Delta \\ \left(M_{f, i-1}-\alpha\right)- & \\ \gamma\left[A A S E_{M_{i}+\Delta}-A A S E_{M_{i}}\right], & \text { otherwise }\end{cases}
$$

where $\alpha$ and $\gamma$ are small positive numbers that satisfy $\alpha<\gamma$, and $M_{i}$ is the integral OCF number at iteration $i$. The average of the accumulated squared-error (AASE) is defined as

$$
A A S E_{M_{i}}=\frac{\sum_{l=0}^{M_{i}} e_{i}^{(l) 2}}{M_{i}+1}
$$

where $e_{i}^{(0)}$ represents $e_{i}$.

Furthermore, $\Delta$ is a positive integer that plays an important role in the pseudo-fractional method. A large value of $\Delta$ yields a fast convergence rate but a large steady-state estimation error. Conversely, a small $\Delta$ results in slow convergence rate but provides a small steady-state estimation error. In order to meet the conflicting requirements of fast convergence rate and small estimation error, the value of $\Delta$ is set to unity in this paper.

Then, the integral OCF number is determined according to

$$
M_{i+1}= \begin{cases}\max \left(\min \left(\left\lfloor M_{f, i}\right\rfloor, M_{\text {max }}\right), 0\right), & \text { if } \zeta \geq \delta \\ M_{i}, & \text { otherwise }\end{cases}
$$

where the operator $\lfloor$.$\rfloor rounds the number to the nearest$ integer, $\delta$ is the threshold parameter that is set to unity in this paper, and $\zeta=\left|M_{i}-M_{f, i}\right|$. It should be noted that $M_{i}$ is updated to satisfy, $0 \leq M_{i} \leq M_{\max }$ where $M_{\max }$ is 
the maximum number of OCFs.

\section{B. Updating Procedure}

From the method proposed above, the updating equation of the proposed algorithm becomes

$$
\hat{\mathbf{w}}_{i+1}=\hat{\mathbf{w}}_{i}+\bar{\mu}_{0} \mathbf{u}_{i}+\bar{\mu}_{1} \mathbf{u}_{i}^{(1)}+\cdots+\bar{\mu}_{M_{i}} \mathbf{u}_{i}^{\left(M_{i}\right)}
$$

where $M_{i}$ is determined by the pseudo-fractional method. If $M_{i}$ is zero, the proposed algorithm will operate as the NLMS algorithm.

The entire procedure of the proposed algorithm is described in Table I.

TABLE I: THE PROPOSED ALGORITHM

\section{Initialization:}

Choose an arbitrary $\hat{\mathbf{w}}_{0}$.

$\alpha, \gamma$ : user defined small positive numbers

$M_{0}=M_{f, 0}=M_{\text {max }}\left(M_{\text {max }}<n\right)$, and $\mu \in(0,2)$.

\section{For each new input $u_{i}$ :}

$e_{i}=d_{i}-\mathbf{u}_{i}^{T} \hat{\mathbf{w}}_{i}$

$\left\|\mathbf{u}_{i}\right\|^{2}=\left\|\mathbf{u}_{i-1}\right\|^{2}+u_{i}^{2}-u_{i-n}^{2}$

$\bar{\mu}_{0}=\frac{\mu e_{i}}{\left\|\mathbf{u}_{i}\right\|^{2}}$

$\hat{\mathbf{w}}_{i+1}^{(1)}=\hat{\mathbf{w}}_{i}+\bar{\mu}_{0} \mathbf{u}_{i}$

$\mathbf{u}_{i}^{(0)}=\mathbf{u}_{i}$

for $k=1,2, \ldots, M_{i}$, do

$$
\begin{aligned}
& \mathbf{u}_{i}^{(k)}=\mathbf{u}_{i-k D}-\sum_{l=0}^{k-1} \frac{\mathbf{u}_{i-k D}^{T} \mathbf{u}_{i}^{(l)}}{\left\|\mathbf{u}_{i}^{(l)}\right\|^{2}} \mathbf{u}_{i}^{(l)} \\
& e_{i}^{(k)}=d_{i-k D}-\mathbf{u}_{i-k D}^{T} \hat{\mathbf{w}}_{i+1}^{(k)} \\
& \bar{\mu}_{k}= \begin{cases}\frac{\mu e_{i}^{(k)}}{\left\|\mathbf{u}_{i}^{(k)}\right\|^{2}}, & \text { if }\left\|\mathbf{u}_{i}^{(k)}\right\| \neq 0 \\
0, & \text { otherwise }\end{cases} \\
& \hat{\mathbf{w}}_{i+1}^{(k+1)}=\hat{\mathbf{w}}_{i+1}^{(k)}+\bar{\mu}_{k} \mathbf{u}_{i}^{(k)}
\end{aligned}
$$

end

$$
\hat{\mathbf{w}}_{i+1}=\hat{\mathbf{w}}_{i+1}^{\left(M_{i}+1\right)}
$$

if $M_{i} \geq \Delta$

$$
M_{f, i}=\left(M_{f, i-1}-\alpha\right)-\gamma\left[A A S E_{M_{i}}-A A S E_{M_{i}-\Delta}\right]
$$

else if

$$
M_{f, i}=\left(M_{f, i-1}-\alpha\right)-\gamma\left[A A S E_{M_{i}+\Delta}-A A S E_{M_{i}}\right]
$$

end

$$
\text { if } M_{i}-M_{f, i} \geq \delta
$$$$
M_{i+1}=M_{i}-1
$$

else if $M_{f, i}-M_{i} \geq \delta$

$$
M_{i+1}=M_{i}+1
$$

end

$M_{i+1}=\max \left(\min \left(M_{i+1}, M_{\max }\right), 0\right)$

end
Remark: The proposed algorithm is designed for stationary environments. However, when the target system is changed, a re-initialization is required in order to achieve fast tracking performance. The proposed algorithm adopts the re-initialization method used in [11], but with modifications. The method of re-initialization is described in Table II.

TABLE II: RE-INITIALIZATION METHOD

$e_{\text {th }}=\mu \sigma_{v}^{2} M_{\text {max }} /(2-\mu)$, flag $=0, e_{\text {avg }}=e_{0}^{2}$

$\lambda, \alpha_{1}, \alpha_{2}$ : user defined

\section{For each $i$ do}

$$
\text { if } \begin{aligned}
e_{i}^{2} & <\alpha_{1} \times e_{t h} \\
\text { flag } & =1
\end{aligned}
$$

else if flag $=1$ and $\alpha_{2} \times e_{a v g}<e_{i}^{2}$

$$
\text { flag }=0, e_{a v g}=e_{i}^{2}, M_{i}=M_{\max }, M_{f, i}=M_{\max }
$$

end

$$
e_{\text {avg }} \leftarrow \lambda e_{\text {avg }}+(1-\lambda) e_{i}^{2}
$$

end

\section{EXPERIMENTAL RESULT}

To illustrate the performance of the proposed algorithm, we carried out computer simulations of a channel estimation. The unknown channels were randomly generated by a moving average model with 32 taps $(n=32)$. The adaptive filter and the unknown channel were assumed to have the same number of taps. The initial number of OCFs for the proposed algorithm, $M_{0}$, was set to 15 , which is the maximum number of OCFs used in the conventional NLMS-OCF algorithm. The input signal $u_{i}$ was generated by filtering a white, zero-mean, Gaussian random sequence through the following systems: $G_{1}(z)=1 /\left(1-0.9 z^{-1}\right), G_{2}(z)=\left(1+0.6 z^{-1}\right) /\left(1+1.0 z^{-1}+0.21 z^{-2}\right)$. The measurement noise $v_{i}$ was added to $y_{i}$ with a signal-to-noise ratio (SNR) of $30 \mathrm{~dB}$, where the $\mathrm{SNR}$ is defined by $10 \log _{10}\left(E\left[y_{i}^{2}\right] / E\left[v_{i}^{2}\right]\right)$ and $y_{i}=\mathbf{u}_{i}^{T} \mathbf{w}$. Additionally, we assumed that the noise variance $\sigma_{v}^{2}$ is known, because this can be estimated during silences in many practical applications [12]-[14]. The mean-squared deviation (MSD), $E\left\|\mathbf{w}-\hat{\mathbf{w}}_{i}\right\|^{2}$, was calculated to indicate the performance of the proposed algorithm and was obtained by ensemble averaging over 100 independent trials. The simulations were performed with $M_{\max }=15, \mu=0.5, D=8$. Furthermore, to check the tracking performance of the proposed algorithm, we suddenly change the coefficients of the unknown filter taps from $\mathbf{W}$ to $-\mathbf{W}$ at time $i=10000$.

\section{A. Performance Comparison}

From Fig. 2 and Fig. 3, we confirm that the NLMS-OCF has a best performance when the number of OCF is decremented from a large number to a small number.

Fig. 4 and Fig. 5 show the MSD curves of the normalized LMS, the conventional APA, the conventional NLMS-OCF, the DS-APA, the DS-N-APA, and the proposed algorithm 
with the input signal generated by $G_{1}(z)$ and $G_{2}(z)$. As shown in the figures, the convergence rate of the proposed algorithm is almost the same as that of the APA, the NLMS-OCF, the DS-APA, and the DS-N-APA. However, the steady-state estimation errors of the NLMS-OCF, the DS-APA, and the DS-N-APA are large, because these algorithms use multiple input vectors even in the steady state. Conversely, the proposed algorithm has the smallest estimation error in the steady state as compared to other members of the APA family.

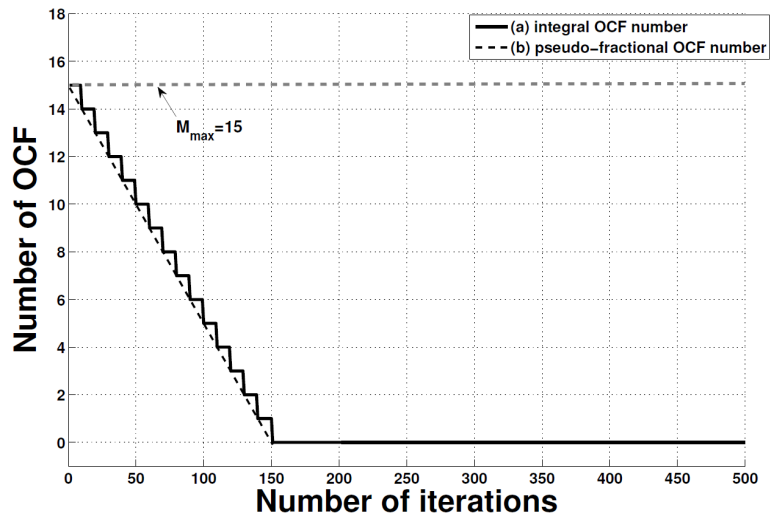

Fig. 2. The number of integral OCF and pseudo-fractional OCF for one trial in the proposed algorithm (The input signal is generated with $G_{1}(z), n=32$, $\mathrm{SNR}=30 \mathrm{~dB})$.

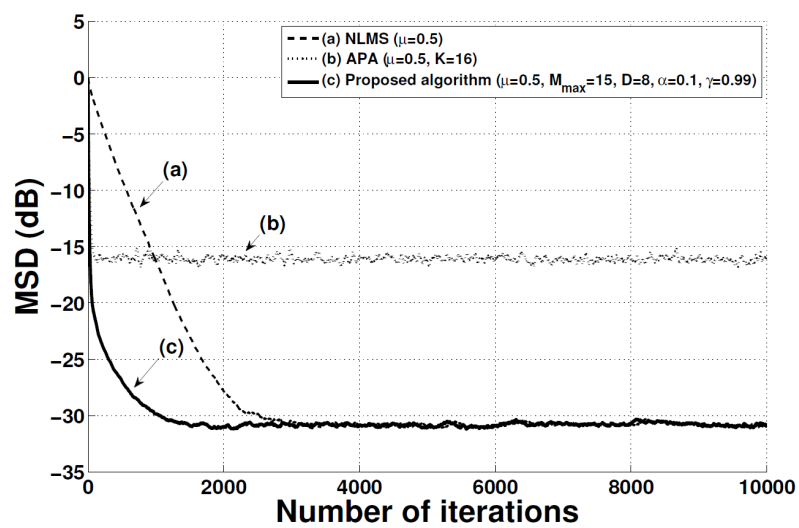

Fig. 3. The MSD curves of the NLMS, the APA, and the proposed algorithm (The input signal is generated with $G_{1}(z), n=32, \mathrm{SNR}=30 \mathrm{~dB}$ ).

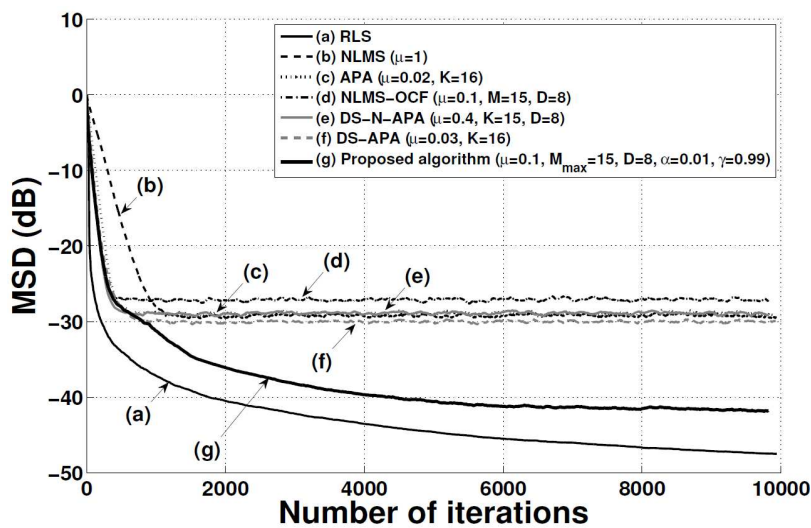

Fig. 4. The MSD curves of the RLS, the NLMS, the APA, the NLMS-OCF, the DS-APA, the DS-N-APA, and the proposed algorithm (The input signal is generated with $\left.G_{1}(z), n=32, \mathrm{SNR}=30 \mathrm{~dB}\right)$.

Fig. 6 shows the tracking capability of the proposed algorithm when the unknown system is suddenly changed. As it is shown, we can confirm that the proposed algorithm keeps the performance for tracking the changed weight without degradation of the convergence rate or the steady-state estimation errors.

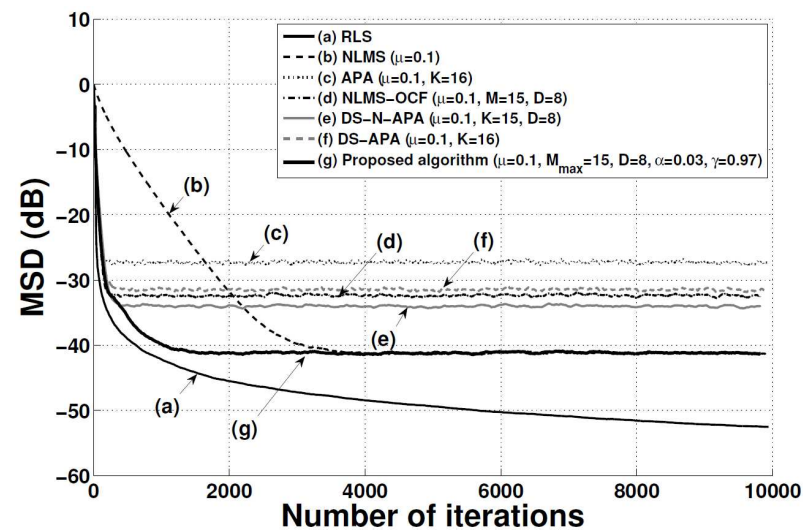

Fig. 5. The MSD curves of the RLS, the NLMS, the APA, the NLMS-OCF, the DS-APA, the DS-N-APA, and the proposed algorithm (The input signal is generated with $\left.G_{2}(z), n=32, \mathrm{SNR}=30 \mathrm{~dB}\right)$.

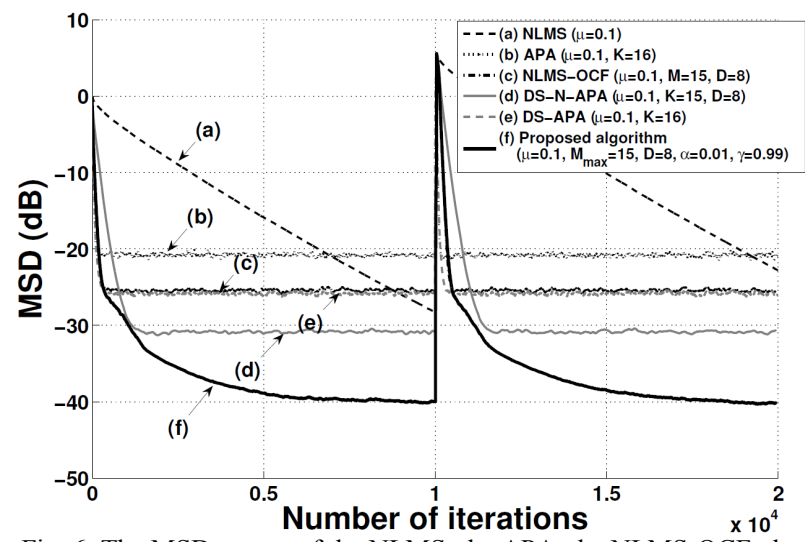

Fig. 6. The MSD curves of the NLMS, the APA, the NLMS-OCF, the DS-APA, the DS-N-APA, and the proposed algorithm (The input signal is generated with $\left.G_{1}(z), n=32, \mathrm{SNR}=30 \mathrm{~dB}\right)$.

Fig. 7 shows the MSD curves of the normalized LMS, the conventional APA, the conventional NLMS-OCF, the DS-APA, the DS-N-APA, and the proposed algorithm with the input signal generated by $G_{1}(z)$ when the SNR is $10 \mathrm{~dB}$.

As shown in the figure, the proposed algorithm has better performance than the existing APAs when the SNR is set lower.

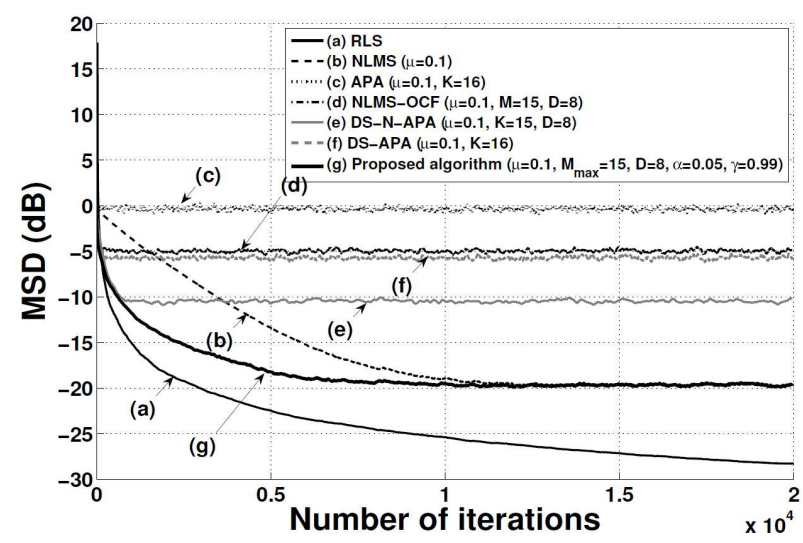

Fig. 7. The MSD curves of the RLS, the NLMS, the APA, the NLMS-OCF, the DS-N-APA, the DS-APA, and the proposed algorithm (The input signal is generated with $\left.G_{1}(z), n=32, \mathrm{SNR}=30 \mathrm{~dB}\right)$. 


\section{B. Computational Complexity}

Table III shows the computational complexity for each iteration of the conventional NLMS-OCF, the DS-N-APA, and the proposed algorithm. The numbers of OCFs for the NLMS-OCF algorithm, the DS-N-APA, and the proposed algorithm are $M, M_{k}$, and $M_{i}$, respectively.

TABLE III: COMPUTATIONAL COMPLEXITY OF THE NLMS-OCF, THE DS-N-APA, AND THE PROPOSED ALGORITHM

\begin{tabular}{|c|c|c|}
\hline & Addition/Subtraction & Multiplication/Division \\
\hline RLS [2] & $n^{2}+3 n$ & $n^{2}+5 n+2$ \\
\hline NLMS [2] & $3 n$ & $3 n+2$ \\
\hline APA [3] & $\left(K^{2}+2 K\right) n+K^{3}$ & $\left(K^{2}+2 K\right) n+K^{3}+K^{2}$ \\
\hline $\begin{array}{l}\text { NLMS- } \\
\text { OCF [5] }\end{array}$ & $\begin{array}{l}2 n+2 \\
+M(M+1) \times(2 n-1) / 2 \\
+M(3 n-1)\end{array}$ & $\begin{array}{l}2 n+4 \\
+M(M+1) \times(2 n+1) / 2 \\
+M(3 n+2)\end{array}$ \\
\hline $\begin{array}{c}\text { DS-N- } \\
\text { APA [8] }\end{array}$ & $\begin{array}{l}(M+1)(n+1)+2 n-1 \\
+M_{k}\left(M_{k}+1\right)(2 n-1) / 2 \\
+M_{k}(3 n-1)\end{array}$ & $\begin{array}{l}(M+1) n+2 n+2 \\
+M_{k}\left(M_{k}+1\right)(2 n+1) / 2 \\
+M_{k}(3 n+2)\end{array}$ \\
\hline $\begin{array}{l}\text { Proposed } \\
\text { Algorithm }\end{array}$ & $\begin{array}{l}2 n+2 \\
+M_{i}\left(M_{i}+1\right) \times(2 n-1) / 2 \\
+M_{i}(3 n-1)+M_{i}+5\end{array}$ & $\begin{array}{l}2 n+4 \\
+M_{i}\left(M_{i}+1\right) \times(2 n+1) / 2 \\
+M_{i}(3 n+2)+M_{i}+4\end{array}$ \\
\hline
\end{tabular}

Fig. 8 shows the accumulated numbers of multiplications. The overall number of multiplications is much lower for the proposed algorithm than for the NLMS-OCF algorithm or the DS-N-APA, because the number of OCFs in the steady state is much smaller for the proposed algorithm than for the NLMS-OCF algorithm or the DS-N-APA.



Fig. 8. Accumulated numbers of multiplications for the RLS, the NLMS, the NLMS-OCF, the DS-N-APA, and the proposed algorithm.

\section{Acoustic Echo Cancellation}

In order to verify the performance of the proposed algorithm for a non-stationary input signal, we also performed an experiment with an input signal of speech sampled at $8 \mathrm{kHz}$ and an acoustic echo path of length $n=1024$. The simulation was performed with $M_{\max }=7, \mu=1, D=4, \alpha=0.001$. Fig. 9 shows the exact impulse response of the room echo path that should be identified. Fig. 10 shows the MSD curves of the APA, the NLMS-OCF with a different $M$, and the proposed algorithm. As shown, we can confirm that the proposed algorithm performs well for a non-stationary signal such as speech.

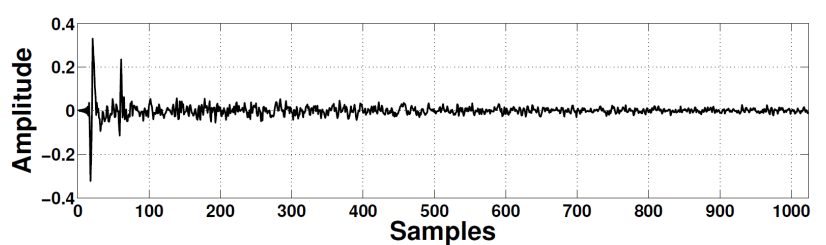

Fig. 9. Acoustic impulse response of a room used in the simulation.

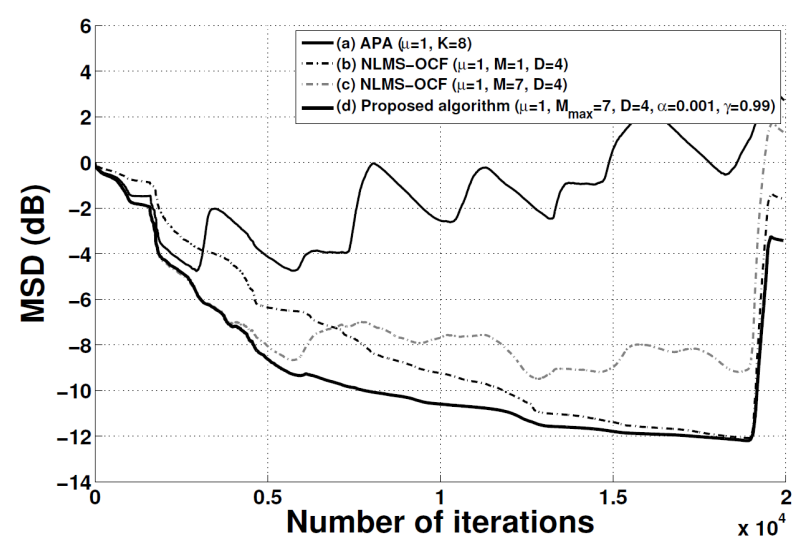

Fig. 10. MSD curves of the APA, the NLMS-OCF, and the proposed algorithm. (The input is a speech signal at $8 \mathrm{kHZ}$ with $n=1024$ and SNR $=$ $30 \mathrm{~dB})$.

\section{CONCLUSION}

This paper has proposed an NLMS-OCF algorithm that adjusts the number of OCFs through a pseudo-fractional method. This method dynamically adjusts the number of OCFs using the proposed adaptation rule and relaxes the constraint that the number of OCFs must be integral. The proposed adaptation rule uses not only an integral OCF number but also a pseudo-fractional OCF number, and it determines the number of OCFs by using the difference between the averages of the accumulated squared-output errors. The proposed method leads to a fast convergence rate, a low steady-state estimation error, and low computational complexity for colored inputs. The experimental results show the improved performance of the proposed algorithm in comparison to the existing family of APAs.

\section{ACKNOWLEDGMENT}

This research was supported by the MSIP (Ministry of Science, ICT and Future Planning), Korea, under the "IT Consilience Creative Program" (NIPA-2014-H0201-14-1001) supervised by the NIPA (National IT Industry Promotion Agency).

\section{REFERENCES}

[1] S. Haykin, Adaptive Filter Theory, 4th ed. Upper Saddle River: Prentice-Hall, 2002.

[2] A. H. Sayed, Fundamentals of Adaptive Filtering, New York: Wiley, 2003.

[3] K. Ozeki and T. Umeda, "An adaptive filtering algorithm using an orthogonal projection to an affine subspace and its properties," Electronics and Communications in Japan, vol. 67-A, no. 5, pp. 19-27, 1984.

[4] S. G. Kratzer and D. R. Morgan, "The partial-rank algorithm for adaptive beamforming," in Proc. SPIE Real-Time Signal Processing VIII, 1985, pp. 9-14.

[5] S. G. Kratzer and A. A. L. Beex, "Normalized LMS algorithm with orthogonal correction factors," in Proc. the Thirty First Asilomar Conference on Signals, Systems, and Computers, 1997, pp. 1670-1673. 
[6] S.-J. Kong, K.-Y. Hwang, and W.-J. Song, "An affine projection algorithm with dynamic selection of input vectors," IEEE Signal Processing Letters, vol. 14, no. 8, pp. 529-532, August 2007.

[7] K.-Y. Hwang and W.-J. Song, "An affine projection adaptive filtering algorithm with selective regressors," IEEE Transactions on Circuits and Systems II: Express Briefs, vol. 54, no. 1, pp. 43-46, Jan 2007.

[8] Y.-Y. Zhu, X. Li, J.-Z. Chen, and Y.-G. Zhu, "The family of affine projection algorithm with dynamic selection of input vectors," in Proc. 2010 International Conference on Wireless Communications and Signal Processing, 2010, pp. 21-23.

[9] Y. Gong and C. F. Cowan, "An LMS style variable tap-length algorithm for structure adaptation," IEEE Transactions on Signal Processing, vol. 53, no. 7, pp. 2400-2407, Jul. 2005.

[10] G. H. Golub and C. F. V. Loan, Matrix Computations, the Johns Hopkins University Press, 1996.

[11] M. Chang, N. Kong, and P. Park, "Variable regularized least-squares algorithm: One step-ahead cost function with equivalent optimality," Signal Processing, vol. 91, no. 5, pp. 1224-1228, May 2011.

[12] N. R. Yousef and A. H. Sayed, "A unified approach to the steady-state and tracking analyses of adaptive filters," IEEE Transactions on Signal Processing, vol. 49, no. 2, pp. 314-324, February 2001.

[13] J. Benesty, H. Rey, L. R. Vega, and S. Tressens, "A nonparametric vss nlms algorithm," IEEE Signal Processing Letters, vol. 13, no. 10, pp. 581-584, October 2006.

[14] M. A. Iqbal and S. L. Grant, "Novel variable step size nlms algorithms for echo cancellation," in Proc. IEEE International Conf. on Acoustics, Speech and Signal Processing, 2008, pp. 241-244.

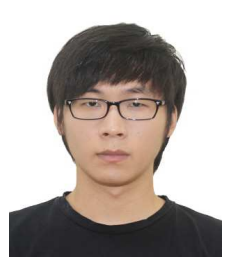

Sang Mok Jung received the B.Sc. and M.Sc. degrees in electrical engineering from Pohang University of Science and Technology (POSTECH), Pohang, Korea, in 2010 and 2012, respectively. Currently, he is a $\mathrm{Ph} . \mathrm{D}$. degree candidate of the division of electrical engineering, POSTECH.

His main research interests include signal processing, adaptive filtering algorithm, image inspections, network programming, and their implementations.

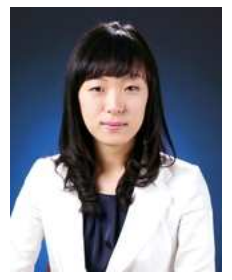

Ji-Hye Seo received the B.Sc. degree in electrical engineering from Pohang University of Science and Technology (POSTECH), Pohang, Korea, in 2010, and the M.Sc. degree in division of IT convergence engineering from POSTECH. Currently, she is a Ph.D. candidate of the division of IT convergence engineering, POSTECH, in 2012.

Her main research interests include signal processing. processing, adaptive filtering algorithm, and image

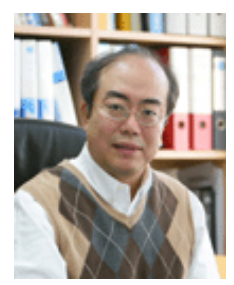

Poo Gyeon Park received the B.S. degree and M.S. degree in control and instrumentation engineering from Seoul National University, Korea, in 1988 and 1990, respectively, and the Ph.D. degree in electrical engineering from Stanford University, Stanford, CA, in 1995.

Since 1996, he has been affiliated with the Division of Electrical Engineering, Pohang University of Science and Technology, where he is currently a professor. His current research interests include robust, LPV, and network-related control theories, delayed systems, fuzzy systems, signal processing, and wireless communications for personal area network (PAN). 